\title{
Effect of preoperative gabapentin and acetaminophen on opioid consumption in video-assisted thoracoscopic surgery: a retrospective study
}

\author{
Robert Qiu ${ }^{1}$, Albert C. Perrino, Jr ${ }^{1,2}$, Holly Zurich ${ }^{1}$, Nitin Sukumar ${ }^{1}$, Feng Dai ${ }^{1}$, Wanda Popescu ${ }^{1,2}$
}

${ }^{1}$ Department of Anesthesiology, Yale School of Medicine, New Haven, CT, USA

${ }^{2}$ Veterans Administration Connecticut Healthcare System, West Haven, CT, USA

\begin{abstract}
Background: Patients undergoing video-assisted thoracoscopic surgery (VATS) are particularly vulnerable to opioid-induced sedation and hypoventilation. Accordingly, reducing opioid consumption in these patients is a primary goal of multimodal analgesic regimens. Although administration of preoperative gabapentin and acetaminophen has been shown to decrease postoperative opioid consumption in other surgeries, this approach has not been studied in VATS lobectomy. Our objective was to examine the impact of the addition of preoperative gabapentin and acetaminophen to a VATS lobectomy multimodal analgesic plan on postoperative opioid consumption, nausea/vomiting, and sedation.

Methods: With IRB approval, we performed a retrospective chart review of patients who underwent VATS lobectomy at a single center between 2015 and 2016 to identify those that received preoperative gabapentin and acetaminophen and those that received neither. Opioid consumption in the first 24 hours postoperatively was converted to oral morphine equivalents (OMEQs). Postoperative sedation was evaluated using Aldrete scores and the percentage of patients requiring antiemetics in the first 24 hours was also examined.

Results: There were 133 patients who were opioid naive: 31 received preoperative gabapentin and acetaminophen and 102 received neither. Median 24 hour postoperative opioid consumption was lower but not statistically significant in the gabapentin and acetaminophen group vs. neither (36 mg vs. $45 \mathrm{mg}, \mathrm{p}=$ 0.08). Notably, there was a change in the distribution of opioid consumption, with no patients in the gabapentin and acetaminophen group requiring more than $200 \mathrm{mg}$ OMEQ in the first 24 hours postoperatively. No significant difference in postoperative nausea/vomiting or sedation was observed.

Conclusions: The addition of preoperative gabapentin and acetaminophen to a VATS lobectomy multimodal analgesic regimen reduces the incidence of high dose postoperative opioid consumption without observed negative side effects.
\end{abstract}

Keywords: acute pain, thoracic surgical procedures, postoperative pain, acetaminophen, gabapentin

\section{Introduction}

Successful management of acute postoperative pain in thoracic surgery is a critical element of the care pathway as it reduces overall morbidity and mortality, particularly that related to pulmonary and cardiac com-

\footnotetext{
Address for correspondence:
}

Robert Qiu, MD

Yale Dept of Anesthesiology

333 Cedar Street, TMP3

New Haven, CT 06510, USA

E-mail: Robert.Qiu@yale.edu plications [1]. However, pain management following thoracic surgery, including video assisted thoracoscopic surgery (VATS), continues to be challenging $[1,2]$. These patients are highly susceptible to opioid-induced over sedation and respiratory depression due to their compromised health status and impaired respiratory function. Accordingly, opioid sparing techniques, such as multimodal analgesia, are advocated $[1,3,4]$. Consensus guidelines issued jointly by the American Pain Society, American Society of Regional Anesthesia, and American Society of Anesthesiologists recommend multimodal analgesia and, specifically for thoracic surgery, suggest NSAIDs, acetaminophen, gabapentin, 
and/or pregabalin [5] as therapeutic agents to reduce opioid consumption.

In non-thoracic surgeries, such as gynecological surgery, gabapentin and acetaminophen administered preoperatively have shown benefit in reducing opioid consumption [6-10]. However, there are no studies to evaluate the impact on postoperative opioid consumption of these medications as part of a multimodal analgesic regimen in patients undergoing VATS procedures. We conducted a retrospective study comparing perioperative opioid consumption in patients undergoing VATS lobectomies who received preoperative gabapentin and acetaminophen (intervention group) versus patients who received neither (control group) as part of a multimodal analgesic program. Additionally, postoperative antiemetic requirement and postoperative sedation scores were compared between the two groups.

\section{Methods}

With IRB approval, we performed a retrospective chart review of patients who underwent VATS lobectomy at a single tertiary center between 2015 and 2016. Patients who received both gabapentin and acetaminophen or neither drug preoperatively were included in the study. Patients who underwent VATS for any other procedures, had chronic pain or received a thoracic epidural were excluded. Data collected included demographic information, ASA physical classification, past medical history, body mass index, and duration of surgery.

The total dose of intraoperative opioids was recorded and converted to oral morphine equivalents (OMEQs) using standard equianalgesic conversion tables [11-14]. Postoperative opioid consumption for the first 24 hours after surgery was also collected and converted to OMEQs. Intraoperative and postoperative non-steroidal anti-inflammatory drug (NSAID) usage was recorded as was intraoperative ketamine administration. As part of our standard practice, all patients received ON-Q ${ }^{\circledR}$ PainBuster ${ }^{\circledR}$ (Halyard Health, Inc., Alpharetta, GA) paravertebral catheters on the surgical side, continuously infusing bupivacaine $0.5 \%$ at $4 \mathrm{ml} / \mathrm{hr}$.

Postoperative antiemetic usage in the first 24 hour period was also collected to assess the incidence of nausea and vomiting. Postoperative sedation was evaluated as part of the Aldrete score collected at the time of arrival in the post anesthesia care unit (PACU).

\section{Statistical analysis}

Patient demographics and case characteristics were summarized among those who received both gabapentin and acetaminophen and among those who received neither. Continuous variables were summarized as means with standard deviations or medians with interquartile ranges, while categorical variables were presented as frequencies with proportions. Statistical comparisons between the two groups were performed using t-tests or Wilcoxon rank sum tests for continuous variables and Chi-square or Fisher's exact tests for categorical variables. Tests were two-sided with a $p<$ 0.05 considered statistically significant. All analysis was conducted using the SAS version 9.4 (SAS Institute, Cary, NC).

\section{Results}

A total of 133 patients met the inclusion criteria: 31 from the intervention group and 102 from the control group. In the intervention group, patients received thirty minutes preoperatively a single oral dose of gabapentin ( 2 patients $300 \mathrm{mg}, 1$ patient $400 \mathrm{mg}$ and 28 patients $600 \mathrm{mg}$ ) and acetaminophen $(975 \mathrm{mg})$. Table 1 shows the demographic information, patient past medical history, and surgical duration. The differences between the two groups were not statistically significant.

Intraoperative medication administration was compared between the intervention group versus the control group in Table 2. Although the mean intraoperative opioid consumption in OMEQs was significantly lower in the intervention group (105.85 $\mathrm{mg}$ versus $125.16 \mathrm{mg}, \mathrm{p}=0.049$ ), the median difference in intraoperative opioid consumption was not statistically significant (100.0 mg versus $106.5 \mathrm{mg}, \mathrm{p}-0.22)$. A significantly larger percentage of patients received intraoperative NSAIDs in the intervention group $(58.84 \%$ versus $30.39 \%, p=0.013)$. The NSAID administration was performed at the end of the surgical procedure, before emergence. There was no statistically significant differences in the percentage of patients receiving intraoperative ketamine $(20.59 \%$ versus $25.81 \%, \mathrm{p}=0.54)$. All patients received intraoperative ondansetron before emergence.

Medication administered postoperatively and Aldrete scores are shown in Table 3. Again, there is a statistically significant decrease in mean postoperative opioid consumption in OMEQs in the first 24 hours following surgery in the intervention group (39.2 mg versus 59.03 $\mathrm{mg}, \mathrm{p}=0.027$ ), with no statistically significant difference in median postoperative opioid consumption. Figure 1 shows the distribution of postoperative opioid consumption, with $0 \%$ of patients in the gabapentin and acetaminophen group requiring high dose opioids (more than $200 \mathrm{mg}$ OMEQs) versus $4 \%$ of patients in the control group. The NSAID and antiemetic administration were not significantly different between the two groups in the first 24 hours postoperatively. The first reported PACU or intensive care unit Aldrete scores were also not significantly different between the two groups. 
Table 1. Demographics, patient, and surgical characteristics

\begin{tabular}{lccc}
\hline Parameters & $\begin{array}{c}\text { Control } \\
(\mathrm{n}=102)\end{array}$ & $\begin{array}{c}\text { Intervention } \\
(\mathrm{n}=31)\end{array}$ & $\mathrm{p}$ \\
\hline Age, years & $66.41( \pm 11.72)$ & $66.32( \pm 9.92)$ & 0.94 \\
Sex, female $(\%)$ & $58(56.86 \%)$ & $16(51.61 \%)$ & 0.61 \\
Race $(\%)$ & & $27(87.10 \%)$ & 1.00 \\
White Non-Hispanic & $87(85.29 \%)$ & $2(6.45 \%)$ & \\
Black & $7(6.86 \%)$ & $2(6.45 \%)$ & 0.89 \\
Other & $8(7.84 \%)$ & $27.09( \pm 4.52)$ & \\
BMI & $27.83( \pm 6.74)$ & $2(6.45 \%)$ & \\
ASA physical status & $5(4.90 \%)$ & $28(90.32 \%)$ & \\
ASA II & $91(89.22 \%)$ & $1(3.23 \%)$ & \\
ASA III & $6(5.88 \%)$ & & \\
ASA IV & & $21(67.74 \%)$ & 0.05 \\
Past medical history $(\%)$ & $49(48.04 \%)$ & $3(9.68 \%)$ & 0.11 \\
Hypertension & $15(14.71 \%)$ & $8(25.81 \%)$ & 0.20 \\
Coronary artery disease & $14(13.73 \%)$ & $21(67.74 \%)$ & 0.63 \\
Diabetes mellitus & $56(54.90 \%)$ & $5.75( \pm 1.69)$ & \\
Hyperlipidemia & $5.93( \pm 1.75)$ & & \\
Surgical duration, hours & & & \\
\hline
\end{tabular}

Results are presented as means $\pm \mathrm{SD}$ or percentage

Table 2. Intraoperative medications

\begin{tabular}{lccc|}
\hline $\begin{array}{l}\text { Intraoperative } \\
\text { medications }\end{array}$ & $\begin{array}{c}\text { Control } \\
(\mathrm{n}=102)\end{array}$ & $\begin{array}{c}\text { Intervention } \\
(\mathrm{n}=31)\end{array}$ & $\mathrm{p}$ \\
\hline $\begin{array}{l}\text { Opioids in OMEQs* } \\
\text { Mean (SD) }\end{array}$ & $125.16( \pm 65.88)$ & $105.85( \pm 39.70)$ & $\mathbf{0 . 0 4 9}$ \\
Median (IQR) & $106.5(88.0-135.0)$ & $100.0(75.0-147.0)$ & 0.22 \\
NSAID (\%) & $31(30.39 \%)$ & $17(54.84 \%)$ & $\mathbf{0 . 0 1 3}$ \\
Ketamine (\%) & $21(20.59 \%)$ & $8(25.81 \%)$ & 0.54 \\
\hline
\end{tabular}

* oral morphine equivalents in $\mathrm{mg}$

Results are presented as means $\pm \mathrm{SD}$, percentage, or median (interquartile range)

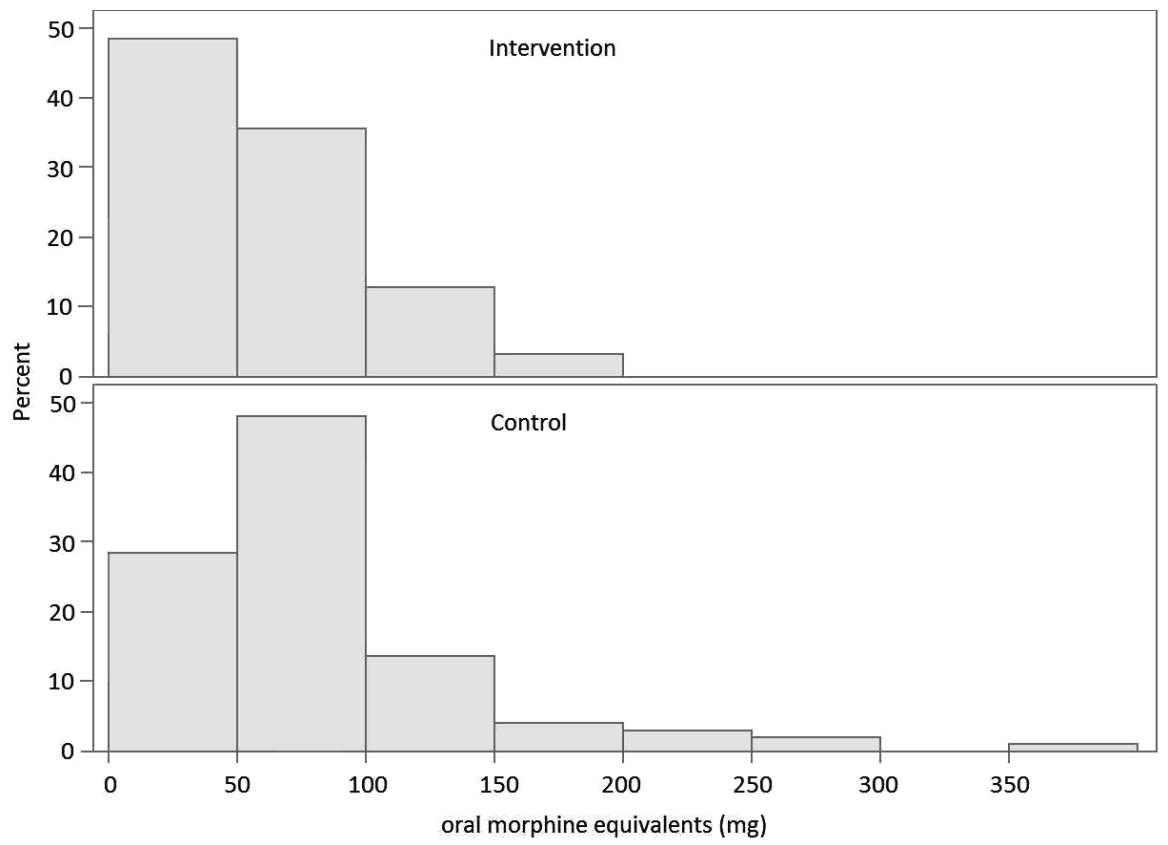

Fig. 1. Distribution of 24-hour postoperative opioid consumption 
Table 3. Postoperative medications administered in the first 24 hours, Aldrete score first reported in PACU or ICU

\begin{tabular}{lccc}
\hline Postoperative & $\begin{array}{c}\text { Control } \\
(\mathrm{n}=102)\end{array}$ & $\begin{array}{c}\text { Intervention } \\
(\mathrm{n}=31)\end{array}$ & $\mathrm{p}$ \\
\hline Opioids in OMEQs* & $59.03( \pm 59.95)$ & $39.20( \pm 36.26)$ & $\mathbf{0 . 0 2 7}$ \\
Mean (SD) & $45.0(21.0-74.5)$ & $36.0(10.0-55.0)$ & 0.08 \\
Median (IQR) & $56(54.90 \%)$ & $19(61.29 \%)$ & 0.53 \\
NSAID (\%) & $31(30.69 \%)$ & $8(25.81 \%)$ & 0.60 \\
Antiemetics (\%) & $8.44( \pm 0.87)$ & $8.52( \pm 0.91)$ & 0.67 \\
Aldrete score & & \\
* oral morphine equivalents in mg & \\
Results are presented as means \pm SD, percentage, or median (interquartile range) &
\end{tabular}

\section{Discussion}

The present study reveals that VATS lobectomy patients who received an analgesic regimen including preoperative gabapentin and acetaminophen exhibited decreased intraoperative and postoperative mean opioid consumption. More importantly, in the intervention group a much smaller percentage of patients required large doses of opioids in the postoperative period, with no patient requiring more than $200 \mathrm{mg}$ OMEQs in the first 24 hours after surgery (Figure 1). These findings support the preoperative oral administration of gabapentin and acetaminophen as part of a multimodal analgesic regimen.

Mechanisms involved in post-thoracic surgery pain are nociceptive and neuropathic in nature and contain both somatic and visceral afferents. The nociceptive somatic afferents are transmitted via the intercostal nerves and are related to rib retraction, muscle splitting, injury to the parietal pleura and chest tube insertion. The nociceptive visceral afferents are transmitted via the phrenic and vagus nerves and are activated by surgical trauma of the bronchi, visceral pleura and pericardium. Furthermore, various inflammatory mediators (prostaglandins, histamine) released as a result of surgery enhance the activity of nociceptors. Ultimately, nociceptive information results in the activation of the neurons in the posterior horn of the spine as well as the central N-methyl-D-aspartate (NMDA) receptors. The neuropathic pain in thoracic surgery is usually the result of injury to the intercostal nerves. It typically manifests as allodynia and hyperalgesia [15]. Additionally, patients undergoing thoracic surgery frequently experience pain to the ipsilateral shoulder. This "referred pain" is caused by the irritation of the visceral pleura from the chest tube and by the stapling of the bronchus.

Due to the multifactorial origin of pain in thoracic surgery, utilizing a combination of analgesic approaches targeting different pathways is a logical step in tackling post lung resection pain. Taking advantage of the additive or synergistic effects of drug combinations on controlling pain can most effectively achieve the goal of reducing both pain scores and opioid consumption $[1,5,8]$. Gabapentin is usually used to treat neuropathic pain and its mechanism of action involves binding to the $\alpha_{2} \delta-1$ and $\alpha_{2} \delta-2$ auxiliary subunits of calcium channels in the central nervous system. Preoperative administration of gabapentin has shown to be effective in the management of acute pain by blocking the development of hyperalgesia and reducing central sensitization $[8,9,16]$. Acetaminophen is a centrally acting analgesic and antipyretic. The mechanism of action is unknown but may involve the modulation of the endocannabinoid system in the brain. Acetaminophen's metabolite appears to inhibit the reuptake of the endogenous cannabinoid by neurons, making it more available to reduce pain [17]. NSAIDs inhibit the COX enzyme preventing inflammation and prostaglandin synthesis [1]. In thoracic surgery, NSAIDs are particularly useful in reducing opioid consumption and treating referred pain to the ipsilateral shoulder. Intravenous ketamine works on blocking pain at the central levels by inhibiting the NMDA receptors and thereby decreasing central sensitization. The most frequently employed intravenous therapy are intravenous opioids which act both peripherally and centrally on mu receptors.

In our study, the intervention group received preoperative gabapentin and acetaminophen, opioids and some patients also received NSAIDs and/or IV ketamine. The control group received IV opioids and some patients received NSAIDs and/or IV ketamine. Furthermore, as part of multimodal analgesia, all patients in both groups also received a continuous paravertebral block ON-Q ${ }^{\circledR}$ PainBuster ${ }^{\circledR}$ (Halyard Health, Inc., Alpharetta, GA) placed under direct vision by the surgeon. Continuous paravertebral analgesia has been shown to be as effective in reducing pain after lung resection surgery as that of thoracic epidurals. As the paravertebral space lacks opioid receptors, only infusion of local anesthetics is administered. The infusion of local anesthetic results in the blockage of both somatic 
and sympathetic afferents from dermatomes above and below the site of the paravertebral catheter.

In the present study, patients in the intervention group had a lower mean opioid consumption both during the intraoperative and postoperative period as compared to the control group. Since the NSAID therapy and the paravertebral catheter were initiated at the completion of surgery, we believe that the lower intraoperative mean opioid consumption is the result of administering preemptive analgesia consisting of gabapentin and acetaminophen. Overall, by targeting multiple pain mechanisms, we have demonstrated success in preventing both excruciating postoperative pain and reducing opioid consumption.

High doses of opioids can result in respiratory depression and opioid-induced sedation which manifest as atelectasis, pulmonary edema, pneumonia, and may lead to reintubation $[1,3,4,18]$. These complications can be particularly detrimental in patients who have undergone thoracic surgery, as these patients have reduced lung function due to chronic obstructive pulmonary disease and lung cancer. Other potential complications related to high dose opioids include an increased risk of deep vein thrombosis, gastrointestinal, infectious, and urinary complications [19]. Furthermore, studies have shown that high dose opioids can increase the risk of recurrence of certain types of cancer which has been attributed to the immunosuppressive effects of opioids [20,21]. Concerningly, high dose opioids have been linked with opioid overdose death in acute and chronic pain. Patients prescribed more than 100 OMEQs/day for acute and postsurgical pain were 6.6 times more likely to die of opioid overdose than those who were prescribed less than 20 OMEQs/day [22, 23].

The literature is heterogeneous on whether preoperative gabapentin has sedating or antiemetic effects $[6,8,16,24]$. However, in this study patients had a similar requirement for rescue antiemetics in the first 24 hours and there were no statistically significant differences in the PACU Aldrete scores between the study groups to suggest increased sedation.

Our study has several limitations. Firstly, this is a retrospective study without the ability to control unknown confounding factors, including which patients received acetaminophen and gabapentin. At our institution several of our anesthesiologists have adopted the practice of administering preoperative acetaminophen and gabapentin, while other anesthesiologists have not. Similarly, other variables in the anesthetic care cannot be controlled without a randomized control trial. Another important limitation of the study is that the overall opioid consumption data showed a large degree of variation between patients as well as a positive skew (Table 3). Due to the fact that the distribution of opioid consumption is nonparametric and that a few patients utilized large doses of opioids which skewed the mean, we compared the medians (using the Wilcoxon rank sum test) as a statistically more robust approach than comparing the means. However, in our study the comparison of the median opioid consumption between the two groups did not reach statistical significance. Additionally, opioid consumption has large interpersonal variability, which increases the required sample size for a study to be adequately powered.

In conclusion, our study showed that VATS lobectomy patients administered preoperative gabapentin and acetaminophen had a reduced incidence of high dose postoperative opioid consumption, an important goal towards reducing morbidity and mortality. No adverse effects, such as increased sedation, were observed with this combination. These findings provide further support for multimodal analgesic regimens to combat the complex pain syndromes following VATS procedures. Additional studies to further validate these results and improve our understanding of perioperative analgesia for VATS procedures are warranted.

\section{Conflict of interest}

Nothing to declare

\section{References}

1. Maxwell C, Nicoara A. New developments in the treatment of acute pain after thoracic surgery. Curr Opin Anaesthesiol 2014; 27: 6-11. doi: 10.1097/ACO.0000000000000029

2. Wildgaard K, Petersen RH, Hansen HJ, Møller-Sørensen H, Ringsted TK, Kehlet H. Multimodal analgesic treatment in video-assisted thoracic surgery lobectomy using an intraoperative intercostal catheter. Eur J Cardiothorac Surg 2011; 41: 10721077. doi: 10.1093/ejcts/ezr 151

3. Joint Commission. Safe use of opioids in hospitals. Sentinel Event Alert 2012; (49): 1-5

4. Jarzyna D, Jungquist CR, Pasero C, Willens JS, Nisbet A, Oakes L, et al. American Society for Pain Management Nursing guidelines on monitoring for opioid-induced sedation and respiratory depression. Pain Management Nurs 2011; 12: 118145. e10. doi: 10.1016/j.pmn.2011.06.008

5. Chou R, Gordon DB, de Leon-Casasola OA, Rosenberg JM, Bickler S, Brennan T, et al. Management of Postoperative Pain: A Clinical Practice Guideline From the American Pain Society, the American Society of Regional Anesthesia and Pain Medicine, and the American Society of Anesthesiologists' Committee on Regional Anesthesia, Executive Committee, and Administrative Council. J Pain 2016; 17: 131-157. doi: 10.1016/j.jpain. 2015.12 .008

6. Durmus M, Kadir But A, Saricicek V, Ilksen Toprak H, Ozcan Ersoy M. The post-operative analgesic effects of a combination of gabapentin and paracetamol in patients undergoing abdominal hysterectomy: a randomized clinical trial. Acta Anaesthesiol Scand 2007; 51: 299-304. doi: 10.1111/j.1399-6576.2006. 01237.x 
7. Alayed N, Alghanaim N, Tan X, Tulandi T. Preemptive use of gabapentin in abdominal hysterectomy: a systematic review and meta-analysis. Obstet Gynecol 2014; 123: 1221-1229. doi: 10.1097/AOG.0000000000000289

8. Arumugam S, Lau CS, Chamberlain RS. Use of preoperative gabapentin significantly reduces postoperative opioid consumption: a meta-analysis. J Pain Res 2016; 9: 631-640. doi: 10.2147/JPR.S112626

9. Seib RK, Paul JE. Preoperative gabapentin for postoperative analgesia: a meta-analysis. Can J Anaesth 2006; 53: 461-469. doi: 10.1007/BF03022618

10. Moon YE, Lee YK, Lee J, Moon DE. The effects of preoperative intravenous acetaminophen in patients undergoing abdominal hysterectomy. Arch Gynecol Obstet 2011; 284: 1455-1460. doi: 10.1007/s00404-011-1860-7

11. Jarlbaek L, Andersen M, Hallas J, Engholm G, Kragstrup J. Use of opioids in a Danish population-based cohort of cancer patients. J Pain Symptom Manage 2005; 29: 336-343. doi: 10.1016/j.jpainsymman.2004.07.010

12. Schneider C, Yale SH, Larson M. Principles of pain management. Clin Med Res 2003; 1: 337-340. doi: 10.3121/cmr.1.4.337

13. American Pain Society. Principles of analgesic use in the treatment of acute pain and cancer pain. 4th ed. American Pain Society; 1999

14. Svendsen K, Borchgrevink P, Fredheim O, Hamunen K, Mellbye A, Dale O. Choosing the unit of measurement counts: the use of oral morphine equivalents in studies of opioid consumption is a useful addition to defined daily doses. Palliat Med 2011; 25: 725-732. doi: 10.1177/0269216311398300

15. Mesbah A, Yeung J, Gao F. Pain after thoracotomy. Bja Education. 2016; 16: 1-7. doi: 10.1093/bjaceaccp/mkv005

16. Mathiesen O, Møiniche S, Dahl JB. Gabapentin and postoperative pain: a qualitative and quantitative systematic review, with focus on procedure. BMC Anesthesiol 2007; 7: 6. doi: 10.1186/1471-2253-7-6
17. Ghanem CI, Pérez MJ, Manautou JE, Mottino AD. Acetaminophen from liver to brain: new insights into drug pharmacological action and toxicity. Pharmacol Res 2016; 109: 119-131. doi: 10.1016/j.phrs.2016.02.020

18. Ruscic KJ, Grabitz SD, Rudolph MI, Eikermann M. Prevention of respiratory complications of the surgical patient: actionable plan for continued process improvement. Cur Opin Anaesthesiol 2017; 30: 399-408. doi: 10.1097/ACO.0000000000000465

19. Cozowicz C, Olson A, Poeran J, Mörwald EE, Zubizarreta N, Girardi FP, et al. Opioid prescription levels and postoperative outcomes in orthopedic surgery. Pain 2017; 158: 2422-2430. doi: $10.1097 /$ j.pain.0000000000001047

20. Oh TK, Jeon JH, Lee JM, Kim MS, Kim JH, Lim H, et al. Association of high-dose postoperative opioids with recurrence risk in esophageal squamous cell carcinoma: reinterpreting ERAS protocols for long-term oncologic surgery outcomes. Dis Esophagus 2017; 30: 1-8. doi: 10.1093/dote/dox074

21. Maher DP, Wong W, White PF, McKenna R Jr, Rosner H, Shamloo B, et al. Association of increased postoperative opioid administration with non-small-cell lung cancer recurrence: a retrospective analysis. Br J Anaesth 2014; 113 Suppl 1: i88i94. doi: 10.1093/bja/aeu192

22. Bohnert AB, Valenstein M, Bair MJ, Ganoczy D, McCarthy JF, Ilgen MA, et al. Association between opioid prescribing patterns and opioid overdose-related deaths. JAMA 2011; 305: 13151321. doi: 10.1001/jama.2011.370

23. Gomes T, Mamdani MM, Dhalla IA, Paterson JM, Juurlink DN. Opioid dose and drug-related mortality in patients with nonmalignant pain. Arch Intern Med 2011; 171: 686-691. doi: 10.1001/archinternmed.2011.117

24. Peng C, Li C, Qu J, Wu D. Gabapentin can decrease acute pain and morphine consumption in spinal surgery patients: A metaanalysis of randomized controlled trials. Medicine 2017; 96. e6463. doi: 10.1097/MD.0000000000006463 